\title{
Visualization of Longitudinal and Transverse Components of Strongly Focused Optical Field by means of Photo-Reactive Azopolymers
}

\author{
A.V. Kharitonov ${ }^{a}$ and S.S. Kharintsev \\ Kazan (Volga Region) Federal University, 420008, 16 Kremlevscaya Str. Kazan Russia
}

\begin{abstract}
Most important problems in modern photonics are fabrication, visualization and characterization of nanomaterials at optical frequencies. A number of optical techniques uses tightly focused laser beams to access longitudinal electromagnetic fields, which are directed towards the wave vector. In this Letter, the distribution of transverse and longitudinal optical fields in tightly focused laser beams, polarized in a new fashion, is investigated. Polarizationdependent fingerprints of transverse and longitudinal optical fields are experimentally captured by means of photoinduced surface deformations in azobenzene polymer thin films.
\end{abstract}

Keywords: radial and azimuthal polarization, longitudinal fields, polarization converter, strong focusing, azobenzene polymer thin films.

Over the last few years, together with the development of three dimensional optical material engineering [1], the designing and manipulation of the spatial structure of optical fields became a topical research area in photonics [2]. In general, an electromagnetic wave structure is characterized by distribution of polarization, amplitude and phase. Until recently, the diversity of polarizations was limited to three types: linear, circular and elliptical. The transformation between these polarizations, as well as them identification is easily accomplished by standard optical instruments such as polarizers, quter- and half-wave plates. The generation of unprecedented polarization states becomes possible due to the development of polarization converters based on multi-element wave-plates [3], metamaterials [4] and plasmonic metasurfaces [5].

This work focuses on investigation of radially and azimuthally polarized laser beams. According to analytical calculations [6], when focusing the radial/azimuthal mode with a high numerical aperture lens (N.A. > 1), a strong longitudinal (towards the wave vector) electric/magnetic field (up to 5 times higher compared to transverse one) arises in the focal region. On the other hand, conventionally polarized light mainly remains the transverse nature provided that light is tightly focused [6]. This unique feature of radial and azimuthal beams make them very attractive for applications, ranging from single molecule spectroscopy [7] and high resolution near-field optical microscopy [8] to optical data storage [9]. In practice, radial and azimuthal modes can be generated through transformation of linearly polarized light by means of special polarization converter. In this Letter we examine two kinds of radial/azimuthal mode converters: polarizer, consisting of four oriented half-wave plates [3], and "s-waveplate" based on metamaterial [4]. Strong focusing the radial and azimuthal modes is

${ }^{a}$ Corresponding author: antonharitonov91@gmail.com 
probed with photoinduced surface deformations in azopolymer thin films $[10,11]$. In order to distinguish the longitudinal and transverse electric field components in the total intensity we apply an optical-field gradient force model [11].

\section{References}

1. W. Cai, V.M. Shalaev, Optical metamaterials: fundamentals and applications. (New York: Springer, 2009)

2. J.B. Pendry, D. Schurig, D.R. Smith, Science. 312, 1780 (2006)

3. R. Dorn, S. Quabis, G. Leuchs, Phys. Rev. Lett. 91, 1 (2003)

4. $\quad$ M. Beresna, M. Gecevicius, P.G. Kazansky, Opt. Mat. Express. 1, 783 (2011)

5. $\quad$ N. Yu, Science 334, 333 (2011)

6. L. Novotny, B. Hecht, Principles of nanooptics. (Cambridge: Cambridge university press, 2006)

7. X. S. Xie, J.K. Trautman, Annu. Rev. Phys. Chem. 49, 441 (1998)

8. L. Novotny, E.J. Sanchez, X.S. Xie, Ultramicroscopy 71, 21 (1998)

9. P. Rochon, J. Gosselin, S. Xie, Appl. Phys. Lett. 60, 4 (1992)

10. Y. Gilbert, R. Bachelot, P. Royer, G.P. Wiederrecht, L. Novotny, Opt. Lett. 31, 613 (2006)

11. S. Bian, J. Appl. Phys. 86, 4497 (1999) 